\title{
Role of Betahistine in the Management of Vertigo
}

\author{
Anirban Biswas ${ }^{1} \quad$ Nilotpal Dutta ${ }^{1}$ \\ ${ }^{1}$ Vertigo and Deafness Clinic, Kolkata, West Bengal, India
}

\begin{abstract}
Address for correspondence Anirban Biswas, MBBS, DLO, Vertigo and Deafness Clinic, BJ 252, Salt Lake, Kolkata 700091, West Bengal, India (e-mail: vertigodeafnesstinnitus@gmail.com).
\end{abstract}

\begin{abstract}
Keywords

- betahistine

- treatment of vertigo

$-\mathrm{H}_{3}$-receptor

- treatment of

Ménière's disease

- betahistine in

Ménière’s disease

Though betahistine is the most commonly prescribed drug for vertigo, there are a lot of controversies on its efficacy as well as its proclaimed mechanism of action. There are authentic studies that have shown it to be no different from a placebo in Ménière's disease. It is often promoted as a vestibular stimulant, but scientific evidence suggests that it is a vestibular suppressant. It is also not very clear whether it is an $\mathrm{H}_{3}$-receptor antagonist as most promotional literature shows it to be, or whether it is an inverse agonist of the $\mathrm{H}_{3}$ receptors. Owing to insufficient data on its efficacy in Ménière's disease, betahistine is not approved by the U.S. Food and Drug Administration (FDA). The much-advertised role of betahistine in augmenting histaminergic transmission and thereby inducing arousal, though beneficial is some ways in the restoration of balance after peripheral vestibulopathy, is yet not without systemic problems, and the pros and cons of histaminergic stimulation in the brain need to be assessed more by clinical studies in humans before imbibing it in clinical practice. The effect of increasing blood flow to the cochlea and the vestibular labyrinth and "rebalancing the vestibular nuclei" (as claimed in some literature) and whether they are actually beneficial to the patient with vertigo in the therapeutic doses are very controversial issues. The mechanism of action of betahistine in vertigo in general and in Ménière's disease in particular is very confusing, and there are too many conjectural and hypothetical, if not controversial issues involved that call for close scrutiny. Everything taken together, betahistine appears to be an unduly hyped-up drug and clinicians need to review the scientific literature available and be convinced about its efficacy and mechanism of action before using it in clinical practice. This review article puts forth some controversial issues and reviews the relevant scientific literature for clinicians to analyze and then take the final call on its clinical use.
\end{abstract}

\section{Introduction}

Betahistine, a structural analogue of histamine, is the one of the most prescribed anti-vertigo drugs today, even though adequate authentic scientific evidence in its favor is lacking. Possibly aggressive marketing and some unscientific promotion may have catapulted it to its present status. Hence, a thorough scientific analysis is warranted. The main problem with betahistine is that reasonably authentic, large, randomized, placebo-controlled, double-blind, multicentric studies proving the efficacy

are generally are not available, whereas less rigorous, observational, and low-quality studies (mostly class 3 ) have shown contradictory results and raised doubts on its clinical efficacy. ${ }^{1}$ It provides symptomatic relief by sedating the vestibular labyrinth and is probably the only nonsedative anti-vertigo drug without any anticholinergic and antidopaminergic effects. It is a vestibular suppressant that does not cause depression of the central nervous system (CNS). ${ }^{2,3}$ Though it is often commercially promoted as a vestibular stimulant, it is not so as per scientific data; it is actually a vestibular suppressant. ${ }^{3}$ There are 
many conflicting views about the mechanism of action (vestibular suppressant or vestibular stimulant?), its clinical efficacy (i.e., whether it is a placebo or has some medicinal/pharmacological effect), the dosage of betahistine, ${ }^{1-5}$ and whether it is a drug only for Ménière's disease or for symptomatic relief for any type of vertigo, etc. This article is an attempt to scan some of the published literature on betahistine and form a consensus on its status as a pharmaco-therapeutic agent in the management of vertigo. Any drug that is used for vertigo will have to serve one or more of the following purposes. It will have to be a drug either for (1) symptomatic therapy, that is, ameliorate the symptoms of vertigo irrespective of the cause, or (2) be a specific therapy for some disease, which is manifested as vertigo, that is, treat or reverse the pathology of the underlying disease where vertigo is the major symptom, or (3) be a drug for prophylaxis of vertigo, that is, prevent or reduce the recurrence of the disease that is causing the recurrent vertigo. Though betahistine is often projected as a drug that serves all the three purposes, yet authentic scientific evidence in its favor is lacking.

\section{Some Contentious Issues about Betahistine}

The reported mechanism of action of betahistine is not consistent in the literature, resulting in confusion in understanding its true pharmacological effects. Hence, though the drug sells very well, the fact remains that most clinicians prescribe it without understanding its mode of action and are possibly influenced by the robust and aggressive marketing strategies and biased propaganda.

Betahistine is a vestibular suppressant ${ }^{3}$ and hence decreases sensory conflicts in the vestibular system when used after a vestibular assault. Hence like all other vestibular sedatives, its use should be restricted to a maximum of 3 to 5 days. Betahistine being a vestibular suppressant, by decreasing vestibular input, suppresses the vestibular system, resulting in delay in vestibular compensation. However, as betahistine is not a CNS depressant and not an anticholinergic drug, the mischief or impediment to vestibular compensation caused by it is expectedly much less as compared with other anti-vertigo drugs.

The much-hyped histamine $\left(\mathrm{H}_{1}\right)$-agonistic action of increasing blood flow to the brain and inner ear with betahistine is pretty weak, as this action is observed only at much higher levels than the therapeutic dose as per different studies. Also, the role of hypoxia in the brain or inner ear and hypoperfusion of the inner ear in the pathophysiology of peripheral vertigo (especially when it is unilateral) are highly questionable issues as vertigo, in general, is not known to be caused by decreased blood flow in the inner ear. Therefore, whether the increase in inner ear blood flow (if at all, in the recommended therapeutic dosage) is of any clinical benefit to the patient of vertigo is very doubtful and possibly inconsequential to say the least.

Betahistine is pharmacologically approved only for Ménière's disease by most pharmacology or medical regulatory bodies but is universally used and promoted for other causes of vertigo also, that is, as a general anti-vertigo drug. It is not a Food and Drug Administration (FDA)-approved drug as there is insufficient evidence of any beneficial effect. ${ }^{6}$ More than 50 years back betahistine was approved by the FDA for sale in the United States, but the approval was withdrawn after 5 years as there was lack of evidence for its efficacy and because the major report of effectiveness contained deficiencies and misrepresentations. ${ }^{7}$

There are wide variations in the dosage of betahistine (24-900 mg/day), and the dose has to be titrated in patients with Ménière's disease. This leads to confusion in the minds of the clinicians as without a definite dosage, use of the drug becomes difficult as clinicians have to try out gradually increasing doses. Usually a drug with a fixed dosage or with a narrow dose range is preferred owing to its ease of usage. Though the pharmacologically approved dosage is only 48 $\mathrm{mg} /$ day, many, if not most, patients especially those with Ménière's disease need much higher doses for any clinically observable benefit with this dose. Most diseases presenting with vertigo, especially Ménière's disease, have phases of waxing and waning and periods of natural remissions and cures; hence, it is impossible to gauge whether a period of remission is actually due to the drug used or due to the natural course of the disease. The observational studies of betahistine in Ménière's disease on a small number of patients in a general ENT (ear-nose-throat) or neurology clinic OPD (many of which are studies sponsored by the marketers of the drug) that abound in the literature basically chronicle the phases of natural remission as this is more common than the phases of acute exacerbation. None of such studies are scientifically authentic class A studies.

Thus, betahistine has not been shown to be better than placebo in many clinical studies, ${ }^{1}$ even at dosages three times the approved dosage. Hence, its clinical efficacy remains at best questionable. Even the Cochrane review $(2001)^{6}$ did not find sufficient evidence of definite beneficial effects with betahistine in Ménière's disease.

\section{Pharmacology}

Betahistine is $\mathrm{N}$-methyl-2- (2-pyridyl) ethylamine dihydrochloride. It is believed to be histamine agonist. It acts as a vasodilator and appears to act directly on the neurons of the vestibular nuclear complex. Betahistine is transformed, mainly at the hepatic level, to aminoethylpyridine, hydroxyethylpyridine, and, finally, to pyridylacetic acid that is excreted in urine.

Symptom-relieving anti-vertigo drugs with proven efficacy and established/undisputed mechanisms of action are mostly antihistamines (centrally acting histamine $\mathrm{H}_{1}$-receptor antagonists). Betahistine, which is a histamine analogue with $\mathrm{H}_{1}$-receptor agonist activity (and $\mathrm{H}_{3}$-receptor antagonist) is also proclaimed to be useful in relieving the symptoms of vertigo, ${ }^{5}$ which means that an antihistaminic and a histamine analogue (which is the opposite of an antihistaminic) both serve the same purpose. This apparently is quite difficult to accept logically, and it means that there may be some lacunae in our understanding of the pathomechanism of vertigo. The modes 
of action are obviously different but two pharmacological agents with completely opposing pharmacological actions serving the same purpose raises doubts on the correctness of the perceived mechanism of action.

A study by Botta et $\mathrm{al}^{8}$ shows that betahistine as well as its metabolite aminoethylpyridine reduces the resting firing rate of the ampulla of semicircular canals; that is, it sedates the resting labyrinth, hence its beneficial effect in vertigo. Betahistine acts on four types of histamine receptors, namely $\mathrm{H}_{1}, \mathrm{H}_{2}, \mathrm{H}_{3}$, and $\mathrm{H}_{4}$. The action of betahistine on $\mathrm{H}_{1}, \mathrm{H}_{2}$, and $\mathrm{H}_{4}$ is weak, but it is very strong on the $\mathrm{H}_{3}$-receptors and most pharmacological effects of betahistine are believed to be due to the effect on $\mathrm{H}_{3}$-receptors. However, the action on $\mathrm{H}_{3}$-receptors is also controversial as betahistine is claimed in some places to be an $\mathrm{H}_{3}$-receptor antagonist ${ }^{4,5}$ and at other places as an $\mathrm{H}_{3}$-inverse agonist,, 3 which indicates that there is a huge confusion in its mechanism of action. There is a lot of difference between an antagonist and an inverse agonist. The entire issue of $\mathrm{H}_{3}$-receptor activity of betahistine is very vague and confusing. In an article by Gbahou et al, ${ }^{9}$ it is stated that "betahistine interacts in vitro with $\mathrm{H}_{3}$ receptors as a potent inverse agonist, a moderate antagonist (Arrang et al, 1985), and a weak agonist. It acts in vivo as a partial inverse agonist to enhance histamine neuron activity." All these only add to the confusion on its mechanism of action. The bottom line is that there is a lot of ambiguity on mechanism of action of betahistine.

\section{Pharmacological Role of Betahistine}

\section{Role as a Vestibular Sedative or Suppressant}

The article on betahistine by Botta et al shows that betahistine and its main metabolite aminoethylpyridine reduce resting discharge rate from the ampulla of the semicircular canals, thus providing a vestibular suppressant effect, thereby leading to a symptomatic relief in vertigo. There are many other articles too that hypothesize that betahistine is a vestibular suppressant or a vestibular sedative as it reduces the discharge rate in the vestibular labyrinth and vestibular nuclei. Timmerman ${ }^{10}$, Lacour, ${ }^{3}$ and even the SERC (betahistine) product manual of Abbott that I could get very clearly state that betahistine is a vestibular suppressant. Lacour ${ }^{3}$ has suggested that it decreases the vestibular input, and Timmerman ${ }^{10}$ has suggested that betahistine causes inhibition of activity in the vestibular nuclei, both of which means that it is a vestibular suppressant.

\section{Role of Enhancing Blood Flow in Inner Ear}

Betahistine is being promoted as an anti-vertigo drug on the logic that it increases blood flow to the brain and inner ear, which is proposed by two mechanisms:

1. By an $\mathrm{H}_{1}$-receptor-mediated agonist action, causing vasodilation ${ }^{5}$; however, the $\mathrm{H}_{1}$-receptor agonistic action of betahistine is pretty weak..$^{1-5}$ Hence, whether this is at all of any clinical consequence at the recommended therapeutic dose is questionable as this effect has been shown in animal studies at very high doses-much higher than the therapeutic dose in humans. It will not be out of place to mention here that the $\mathrm{H}_{3}$ antagonistic effect of betahistine possibly augments the $\mathrm{H}_{1}$ agnostic effect of betahistine by some complex mechanism and so some $\mathrm{H}_{1}$ agnostic effect, i.e., increased microcirculation may be indirectly achieved by betahistine in spite of the very weak effect of betahistine on the $\mathrm{H}_{1}$ receptors.

2. By virtue of its $\mathrm{H}_{3}$-antagonistic action or its so-called inverse agonist action (?), betahistine increases histamine release from the histaminergic nerve endings. This histamine is hypothesized to stimulate $\mathrm{H}_{1}$-receptors, resulting in vasodilatory effects in the inner ear.

However, such an action of betahistine is not easy to understand and accept logically, as it is difficult to increase only the cochlear blood flow without increasing the blood flow in other organs. ${ }^{4}$ It is hard to comprehend what is so very unique anatomically and physiologically in the cochlea that betahistine will increase blood flow only in the cochlea (or for that matter in the inner ear) but not in other organs. Moreover, there are other questionable issues that crop in here such as (1) there is no scientific evidence that all peripheral vertigo including Ménière's disease is primarily caused by hypoperfusion of the inner ear and (2) even if increase in blood flow is beneficial in stopping vertigo, other agents that are known to augment blood flow, such as aspirin, clopidogrel, and xanthine nicotinate, should be equally, if not more effective in increasing blood flow and enhancing micro-circulation and agents that inhibit blood flow should cause vertigo. This is possibly not true either.

\section{Role of Enhancing Arousal and Acceleration of Vestibular Compensation}

Betahistine is claimed to enhance arousal and accelerate vestibular compensation as there has been a consensus on the relevance of vestibular compensation in patients of vertigo especially those of peripheral origin. However, this action of betahistine has been shown in experimental animals (cats) at high doses ( $100 \mathrm{mg} / \mathrm{kg}$ vs. human dose of $24 \mathrm{mg} /$ day equivalent to $0.4 \mathrm{mg} / \mathrm{kg}$ in a $60-\mathrm{kg}$ person). ${ }^{11}$ The mechanism of compensation is said to be by "rebalancing the vestibular nuclei" and by inducing arousal.

It is difficult to understand how a medication can improve rebalancing the vestibular nucleus when it is only used in Ménière's disease (or even in other cases of vertigo) and not in any other condition in which CNS adaptation is required. ${ }^{4}$ The issue of inducing arousal is undisputedly a very desirable effect as the opposite; that is, drowsiness and CNS depression are known to jeopardize central vestibular compensation. However, drugs such as caffeine, the most widely used psychoactive compound, which promotes wakefulness by blocking adenosine $A_{2 A}$-receptors $\left(A_{2 A} R s\right)$ in the brain and possibly acts on the nucleus accumbens, should serve this purpose much better. However, drugs that promote wakefulness are naturally expected to cause sleeplessness that is not known to be caused by betahistine in the doses at which it is commonly prescribed. Therefore, here again this proposed effect of betahistine is suspect at least at recommended therapeutic doses. 
Studies in experimental animals have shown the involvement of histaminergic system in the vestibulohypothalamo-vestibular loop and researchers have hypothesized a possible role of betahistine in effecting arousal that promotes vestibular compensation and in the control of vestibular response after a vestibular assault. Electrophysiological recordings have reportedly shown that unilateral and bilateral stimulation of the labyrinth in which there is asymmetrical vestibular nuclei activity (as happens after a peripheral vestibular lesion) triggers an increase in histamine release in the vestibular nuclei and associated structures (inferior olive, autonomic nuclei) and the tuberomammillary of the posterior hypothalamus (the portion of the brain involved in effecting arousal) in healthy rats. ${ }^{12-15}$ This may have therapeutic implications, and if it induces arousal in humans at the recommended therapeutic doses of betahistine, it is a welcome therapeutic measure. However, currently these experimental findings are all based on animal studies and are possibly only at the laboratory level now. A lot of it is hypothetical, and extrapolation to humans at this stage may not be prudent at the present state of knowledge. There is no relevant human study that the authors came across, nor is there any indication whether the effect of histaminergic stimulation occurs in the recommended therapeutic dosage of betahistine in humans. The actual mechanism of betahistine showing any beneficial effect in restoration of balance in humans by effect of betahistine in the vestibulo-hypothalamo-vestibular loop is basically conjectural now. It needs to be confirmatively ascertained whether in the therapeutic dose of betahistine in humans there is any positive effect induced either in arousal or in vestibular recovery (or for that matter "control of vestibular response") through involvement of the histaminergic system in the vestibulo-hypothalamo -vestibular loop before this aspect is capitalized upon for promoting betahistine in humans. The effects of histamine in the brain have been extensively studied. The interested readers are referred to an article by Passani et al. ${ }^{16}$ It states that "brain histamine promotes wakefulness and orchestrates disparate behaviors and homeostatic functions. Recent evidence suggests that aberrant histamine signaling in the brain may also be a key factor in addictive behaviors and degenerative disease such as Parkinson's diseases and multiple sclerosis." It is also reported that histaminergic stimulation is also involved in the appetitive, food anticipatory responses, and in food consumption, and it has been suggested that it may have an important role in abnormal appetites not only for food but also for alcohol consumption and substances of abuse. ${ }^{16,17}$ Therefore, there is much more to stimulating the histaminergic system in the brain, and it is not just as simple as enhancing arousal only, and by extrapolating the logic thereby stimulating vestibular compensation. New paths should be very cautiously treaded, especially in the medical field. Clinicians should be very discerning and not be influenced by a one-line picked up from some scientific article (often out of context) by some pharma company for promoting betahistine or for that matter any other medicinal agent. It is true that stimulation of the histaminergic system in the brain promotes wakefulness, but should it be the reason enough to try so in all patients with vertigo, considering its systemic effects is highly debatable and may not be conducive to the patient's general health and the clinician's objectives of treatment?

\section{Drug or Placebo?}

The clinical studies evaluating the efficacy of betahistine in Ménière's disease have not shown it to be superior to placebo. The BEMED (the medical treatment of Ménière's disease with betahistine $)^{1}$ trial was a multicentric, double-blind, randomized, placebo-controlled trial, and was published in the British Medical Journal in January 2016. The results of this trial showed that the incidence of attacks related to Ménière's disease did not differ between the three treatment groups: (1) the low-dose betahistine dose of $48 \mathrm{mg} /$ day, (2) high-dose betahistine of $144 \mathrm{mg} /$ day, and ( 3 ) placebo ( $p=0.759$ ). This study showed that placebo has the same effect as betahistine even at pretty high doses of betahistine in Ménière's disease even after 9 months of treatment. This is an authentic evidence on the lack of clinical efficacy with betahistine in Ménière's disease. ${ }^{1}$ A Cochrane review published in $2001^{6}$ contained a systematic review of seven selected randomized controlled trials (involving 242 patients) treated with betahistine versus placebo in Ménière's disease. It concluded that there are insufficient data on the efficacy of betahistine in Ménière's disease. The results of several other studies too endorse the findings that the efficacy of betahistine in Ménière's disease is questionable. A study ${ }^{18}$ evaluated clinical benefits and side effects of high dosages (288-480 mg/day) of betahistine in patients with severe Ménière's disease. It was observed that the drug was only effective at high doses. ${ }^{18}$ This means that if $16 \mathrm{mg}$ tablets are used, then 18 to 30 tablets of betahistine per day would be required, which is not very practicable as compliance would be an issue with many patients. Some authorities promote even higher doses for which even 50 or 60 tablets per day would be required. It would not be out of place here to mention that a chemical agent is now being tried to be combined with betahistine to improve its bio-availability such that smaller doses work, but that too is possible in the experiential stage now.

\section{A Review of a Few Often-Quoted Studies Conducted on Betahistine}

1. One observational placebo-controlled study by Redon et $\mathrm{al}^{19}$ report the influence of betahistine dihydrochloride on vestibular compensation in humans. The study was conducted on 16 patients with Menière's disease who underwent a curative unilateral vestibular neurectomy (UVN) by retrosigmoid approach for therapeutic reasons. All 16 patients had a postoperative bithermal caloric test evidence of a pure unilateral vestibular deficit (complete areflexia on the operated side). Eight of these 16 patients had betahistine (48 mg/day) and 8 had placebo for 3 months post-surgery. The authors report 
that time to recovery was reduced for most of the static postural, oculomotor, and perceptive symptoms tested with laboratory equipment, as well as for the patients' self-evaluation of stability in the eight patients who had betahistine as compared with the eight patients of the placebo group. The study reports that in self-evaluation of stability, the patients under betahistine treatment reported being stable after 5 weeks as compared with 9 weeks for the placebo group. The researchers conclude that betahistine dihydrochloride facilitates functional recovery after unilateral vestibular loss and speculate that the effects of betahistine on vestibular compensation may be related to the action of betahistine at the level of the vestibular nuclei by "rebalancing the neural resting activity between the lesioned and healthy sides."

Author's view: The study group is too small to be indicative of any trend, and whether the mechanism of how betahistine is at all responsible for an early functional recovery is vague and at best speculative. It is not comprehensible how betahistine can "rebalance the neural resting activity of the vestibular nuclei between the lesioned and healthy sides." Even if so (for argument sake), why should such rebalancing of resting neural activity be limited only to the vestibular system? The term "rebalancing" is very vague and appears to be more of a promotional gimmick rather than a scientific entity. All mechanisms of action for betahistine suggested are at best conjectural and prefixed by terms such as "may" and "possibly." There is no scientific evidence available to label it as a "vestibular stimulant." Betahistine is not a CNS depressant, true, but that does not mean that it is a vestibular stimulant. This is stretching imagination too very far. Even if there is increase in histamine release in the vestibular nuclei of the lesioned side, we do not know whether that facilitates recovery in any way or whether that even decreases recovery, as histamine has never been proved to be a repairer of neural tissue or enhancer of neural function. Moreover, there is no definite evidence to show that there is increased histamine release in the vestibular nuclei of the lesioned side or even at the tuberomammillary nucleus at the recommended therapeutic dose in humans.

2. A review article by $\operatorname{Ramos}^{20}$ observes that betahistine is effective and safe in the treatment of Ménière's disease, benign paroxysmal positional vertigo (BPPV), vestibular neuronitis, and other types of peripheral vertigo as demonstrated by clinical studies and meta-analyses. The researchers have concluded that efficacy and safety of betahistine have been demonstrated in numerous clinical trials but have very clearly mentioned that "the precise mechanism of action of betahistine is still not completely understood, though the clinical experience demonstrated the benefit of betahistine in different types of peripheral vertigo."

Author's view: This is as good as promoting any placebo for any disease. Peripheral vertigo is in most cases a self-limiting disorder and the symptomatic relief obtained with betahistine or for that matter any other vestibular suppressant is not disputed. In BPPV, the researchers have mentioned use of betahistine "primarily as a coadjuvant of vestibular compensation for cases with recurrent disease." This logic too is scientifically untenable because vestibular compensation is not required in BPPV as there is no vestibular weakness in BPPV. Also, BPPV is very well treatable by the correct maneuvers only and there is no question of any coadjuvant therapy at all. In the rare cases of post-BPPV syndrome that may be psychogenic in origin or due to the postmaneuver utricular overload, there is no logical role of betahistine. This is usually treated with a short course of benzodiazepines or anxiolytics or antidepressants and sometimes along with a vestibular sedative, but the latter is also usually not required. 3. An article by Lacour ${ }^{3}$ discusses probable mechanisms for role of betahistine in reducing vertigo due to Ménière's disease and other etiologies and enhancing vestibular compensation. This is one of the most comprehensive articles on betahistine and has been referenced several times in this review article as well as in most scientific articles related to betahistine. The message conveyed in this article is that betahistine efficacy can be explained by mechanisms targeting the histamine receptors $\left(\mathrm{H}_{1}, \mathrm{H}_{2}, \mathrm{H}_{3}, \mathrm{H}_{4}\right)$ at three different levels: (1) the vascular tree, with an increase in cochlear and vestibular blood flow involving the $\mathrm{H}_{1}$-receptors; (2) the CNS, with an increase in histamine turnover implicating the $\mathrm{H}_{3}$-receptors, and (3) the peripheral labyrinth, with a decrease in vestibular input implying the $\mathrm{H}_{3}-$ and $\mathrm{H}_{4}-$ receptors. The article contends that clinical data from vestibular loss patients show the impact of betahistine treatment for the long-term control of vertigo, improvement of balance, and quality of life can be explained by the above-mentioned three mechanisms of actions, provided it is used in the right dose and for the right duration. It also states that though antihistaminics are most commonly used in the medical treatment of vertigo, yet because of the sedation induced, they are detrimental for the recovery process whereas betahistine has no sedative effects and hence is more suited for the treatment of Menière's disease and patients with vertiginous syndromes of peripheral origin.

Author's view: Most issues presented in this article have been analyzed in the review. The researcher's contention that as betahistine does not have a sedative effect, it is better placed than the antihistaminics for symptomatic treatment of vertigo is acceptable and undeniable. However, it is reiterated that betahistine not having a sedative effect does not make it a vestibular stimulant as very often claimed by the pharmaceutical companies marketing the drug. As it is not a CNS depressant, it does not induce sedation such as the antihistaminic anti-vertigo drugs, but as stated in the article, it "decreases vestibular input"; hence, it suppresses the vestibular labyrinth or nuclei and is a vestibular sedative and not a vestibular stimulant. The implications of being a vestibular sedative and its effect on vestibular compensation are well known and established. Any vestibular suppressant will automatically reduce the sensory conflicts in the vestibular system and thereby jeopardize or inhibit compensation. The issue of increasing cochlear and vestibular blood flow by its $\mathrm{H}_{1}$-receptor agnostic action too has been 
discussed already. To repeat again, first of all, the $\mathrm{H}_{1}$-receptor agnostic action of betahistine is very mild and is possibly not of much clinical significance in the recommended therapeutic dose, and second, there is no evidence that all peripheral vestibular disorders including Ménière's disease are due to hypoxia of the inner ear or brain and so increasing cochlear and vestibular blood flow in all patients with vertigo, even if achieved by betahistine in the recommended therapeutic dosage (which again is questionable), may not be a clinically beneficial pharmacological action. The issue of increased histamine turnover by betahistine at the recommended therapeutic dosage and its benefit in humans, if any, is at best conjectural at the present state of knowledge. However, betahistine-induced upregulation of histamine possibly induces a general brain arousal favoring sensorimotor activity as proposed by the researcher, and this may enhance vestibular compensation. It is known that vestibular compensation is hampered by sedation but how much it is enhanced by augmenting brain arousal is not known with certainty, but if so, then drugs such as caffeine should also help.

Therefore, after reviewing all this literature, it is difficult to authentically prove any beneficial effects of betahistine. The supportive scientific logic in its support is suspect, and proposed mechanism of action is confusing and vague and clinical implications are at best conjectural.

\section{Controversies Related to Dosage and Duration of Therapy}

Betahistine is available as:

- Dihydrochloride

- Mesylate

\section{Dose at which Betahistine Is Used Shows Wide Variations}

The hydrochloride salt is more common and is prescribed in a wide range of dosage 24 to $900 \mathrm{mg} /$ day although the recommend therapeutic dose is $48 \mathrm{mg} /$ day. However, some centers promote 600 to $900 \mathrm{mg} /$ day or even more in Ménière's disease as the drug is ineffective in many cases at the recommended therapeutic dosage. This raises a question that whether the cases where the vertigo is symptomatically relieved at the recommended therapeutic dose are a natural recovery or is the recovery due to the effect of the drug. Most cases of peripheral vertigo are self-limiting, and natural recovery is very common in vertigo.

\section{Duration of Therapy}

The duration for which the drug can be prescribed is controversial and doubtful as there is lack of clarity on its use and mechanism of action. If betahistine is used as a vestibular suppressant and only for symptomatic relief of vertigo, the duration should be limited to a maximum of 3 to 5 days and not more than that just like any other vestibular sedative. However, here the question is that if symptomatic relief is the purpose, drugs, such as dimenhydrinate or meclizine or even prochlorperazine or a combination of a low fixed dose of dimenhydrinate and cinnarizine, are possibly better choices. If the clinician still wants to use betahistine for symptomatic relief, on account of it being a non-CNS depressant, nonanticholinergic, nondopaminergic, and noncalcium channel blocker drug that are obvious virtues of the drug, the dose should be at least 96 or $144 \mathrm{mg}$ daily (if not more) instead of the 24 to $48 \mathrm{mg}$ daily dose as recommended and in four instead of three divided doses as the mean plasma elimination half life is just 3 to 4 hours. This of course is the authors' personal experience. The dosage in confirmed Ménière's disease is completely controversial. First question is whether betahistine is at all of any help in Ménière's disease in the recommended dosage, as the published authentic literature ${ }^{1}$ says it is not and that it is merely a placebo. Second question is whether all patients with Ménière's disease really require a long continued prophylactic treatment, as in most cases of Ménière's disease the attacks are usually once in a few months or once in several weeks and in all such cases just aborting the occasional attacks suffices and long continued prophylactic/preventive therapy is not required. It is only in cases in which there are very frequent attacks does the question of long-term prophylactic therapy arise. In such cases, there may be some scope of using betahistine on a long-term basis, but the dose needs to be titrated in each individual case. The starting dose may be 72 to 96 $\mathrm{mg} / \mathrm{day}$, and if that does not control symptoms, the dosage can be gradually increased to 248 or $480 \mathrm{mg} /$ day for several months, ${ }^{6}$ but the basis of this dosage and the outcome are not on solid authenticated scientific rationale; hence, many clinicians rely more on diuretics, such as acetazolamide, tiamterene, hydrochlorthiazide, and spironolactone, instead of betahistine for prophylactic treatment of Ménière's disease. There is a lot of natural waxing and waning in the course and severity of Ménière's disease, and the phase of very frequent attacks usually does not persist for more than a few weeks at a stretch; hence, it is impossible to confirmatively know whether there is a natural recovery or is the recovery (if at all) is due to the high dose of betahistine.

\section{Adverse Effects}

Various allergic and skin-related side effects and hypersensitivity reactions such as tingling, numbness, burning sensation, and respiratory distress can occur due to increased histamine level. Betahistine should be used with caution in asthmatics and peptic ulcer patients as it is a mild $\mathrm{H}_{2}$-agonist and can augment gastric secretion. However, as already stated, the $\mathrm{H}_{1}$ and $\mathrm{H}_{2}$ effects are so mild that this probably is of negligible clinical impact. Compared with all other anti-vertigo drugs, betahistine has the least adverse effects and is a safe drug even at reasonably high doses.

Systemic administration of betahistine causes peripheral vasodilatation and a fall in systemic blood pressure; hence, it should be used with caution when prescribed at very high doses. 


\section{Summary}

In summary, betahistine appears to offer some symptomatic relief in vertigo as it is a vestibular suppressant as per scientific literature, ${ }^{3}$ and clinical experience also suggests that betahistine offers some palliative effect in patients with mild to moderate vertigo, especially in patients without much of vegetative symptoms. It is devoid of major adverse effects and is more or less a safe drug with no anticholinergic, antidopaminergic, and antiserotonergic properties and is not a CNS depressant. It may be having some beneficial effects in Ménière's disease but only at very high doses that are much higher than the recommended therapeutic dosage. At commonly prescribed doses, it has been established to be no better than a placebo. It is a common experience that some patients with Ménière's disease and even patients with other causes of vertigo do well when on betahistine, but whether it is due to betahistine or due to the natural course of the disease is debatable. Ménière's disease has a natural history of waxing and waning, and the disease has phases of heightened activity and inactivity when there are no attacks. In most cases, there is a phase of burnout after which there are no episodes of head spinning; hence, gauging the success of pharmacotherapy in Ménière's disease is also not easy. However, there is no denying that it is a much hypedup anti-vertigo drug without solid scientific evidence of its much-advertised beneficial effects. Its pharmacological mechanism of action in vertigo is confusing and controversial and is very difficult to comprehend; hence, clinicians should use it with scientific discretion and clinical logic based on authentic scientific studies and not be influenced by the very aggressive propaganda with which it is marketed.

\section{References}

1 Adrion C, Fischer CS, Wagner J, Gürkov R, Mansmann U, Strupp M; BEMED Study Group. Efficacy and safety of betahistine treatment in patients with Meniere's disease: primary results of a long term, multicentre, double blind, randomised, placebo controlled, dose defining trial (BEMED trial). BMJ 2016;352:h6816

2 Lacour M, Sterkers O. Histamine and betahistine in the treatment of vertigo: elucidation of mechanisms of action. CNS Drugs 2001;15(11):853-870

3 Lacour M. Betahistine treatment in managing vertigo and improving vestibular compensation: clarification. J Vestib Res 2013;23(3):139-151

4 Hain TC. Serc (Betahistine). Available at: https://www. dizziness-and-balance.com/treatment/drug/serc.html. Accessed October 13, 2018
5 Hain TC, Uddin M. Pharmacological treatment of vertigo. CNS Drugs 2003;17(2):85-100

6 James AL, Burton MJ. Betahistine for Menière's disease or syndrome. Cochrane Database Syst Rev 2001;(1):CD001873

7 Sampson WI. Homeopathic vs conventional treatment of vertigo. Arch Otolaryngol Head Neck Surg 2003;129(4):497; author reply 498

8 Botta L, Mira E, Valli S, et al. Effects of betahistine and of its metabolites on vestibular sensory organs. Acta Otorhinolaryngol Ital 2001;21(3 Suppl 66):24-30

9 Gbahou F, Davenas E, Morisset S, Arrang JM. Effects of betahistine at histamine $\mathrm{H} 3$ receptors: mixed inverse agonism/agonism in vitro and partial inverse agonism in vivo. J Pharmacol Exp Ther 2010;334(3):945-954

10 Timmerman H. Pharmacotherapy of vertigo: any news to be expected? Acta Otolaryngol Suppl 1994;513:28-32

11 Tighilet B, Leonard J, Lacour M. Betahistine dihydrochloride treatment facilitates vestibular compensation in the cat. J Vestib Res 1995;5(1):53-66

12 Serafin M, Khateb A, Vibert N, Vidal PP, Mühlethaler M. Medial vestibular nucleus in the guinea-pig: histaminergic receptors. I. An in vitro study. Exp Brain Res 1993;93 (2):242-248

13 Takeda N, Morita M, Kubo T, et al. Histaminergic projection from the posterior hypothalamus to the medial vestibular nucleus of rats and its relation to motion sickness. In: Graham MD, Kemink JL, eds. The Vestibular System: Neurophysiologic and Clinical Research. New York, NY: Raven Press; 1987:571-580

14 Jaju BP, Wang SC. Effects of diphenhydramine and dimenhydrinate on vestibular neuronal activity of cat: a search for the locus of their antimotion sickness action. J Pharmacol Exp Ther 1971;176(3):718-724

15 Kirsten EB, Sharma JN. Microiontophoresis of acetylcholine, histamine and their antagonists on neurones in the medial and lateral vestibular nuclei of the cat. Neuropharmacology 1976;15(12):743-753

16 Passani MB, Panula P, Lin JS. Histamine in the brain. Front Syst Neurosci 2014;8:64

17 Torrealba F, Riveros ME, Contreras M, Valdes JL. Histamine and motivation. Front Syst Neurosci 2012;6:51

18 Lezius F, Adrion C, Mansmann U, Jahn K, Strupp M. High-dosage betahistine dihydrochloride between 288 and $480 \mathrm{mg} /$ day in patients with severe Menière's disease: a case series. Eur Arch Otorhinolaryngol 2011;268(8):1237-1240

19 Redon C, Lopez C, Bernard-Demanze L, et al. Betahistine treatment improves the recovery of static symptoms in patients with unilateral vestibular loss. J Clin Pharmacol 2011;51(4):538-548

20 Ramos Alcocer R, Ledezma Rodríguez JG, Navas Romero A, et al. Use of betahistine in the treatment of peripheral vertigo. Acta Otolaryngol 2015;135(12):1205-1211 